\title{
Pulse propagation in a randomly perturbed ocean: Single pulse statistics
}

\author{
Werner E. Kohler \\ Department of Mathematics, Virginia Polytechnic Institute and State University, Blacksburg, Virginia 24061 \\ (Received 14 September 1979; accepted for publication 15 July 1980) \\ A statistical theory of broadband single pulse propagation in a random ocean is presented. The mutual \\ coherence function of the received signal is derived using an analysis based upon coupled mode theory. As \\ propagation range increases, the combined effects of modal dispersion and random fluctuations spread the \\ pulse and decompose it into a series of multiple arrivals.
}

PACS numbers: 43.30.Bp, 43.30.Gv, 43.60.Cg

\section{INTRODUCTION}

Theories of acoustic signal propagation that view the ocean as a randomly perturbed medium are becoming increasingly successful in accounting for experimental observations, ${ }^{1-7}$ with the work of Flatte, Dashen, Munk, and Zachariasen ${ }^{1}$ being particularly notable. Most of the work to date has dealt with $\mathrm{cw}$ propagation. How ever, pulse propagation has also been studied and the basic physical mechanisms underlying both problems are well understood.

The aim of this paper is to analyze the problem of pulse propagation in a random ocean using a normal mode decomposition. The approach combines features of the work of Porter and Leslie ${ }^{8,9}$ who have used nor mal mode theory to study pulse propagation in a nonrandom channel with the work of Dozier, Tappert, ${ }^{6,7}$ and other ${ }^{4}$ who have used normal mode analysis and the ensuing coupled mode equations to study $\mathrm{cw}$ propagation in a random ocean. The use of Fourier transforms and normal mode decomposition introduces the effect of the sound speed profile (including bottom effects if relevant) into the analysis in a direct and systematic way.

Sections I and II will be devoted to formulating the problem and deriving an asymptotic expression for the mutual coherence function for the complex pressure. Only a brief outline of the stochastic analysis will be given since the theory is well documented elsewhere. ${ }^{10-12}$ Section III will develop some physical implications of the results.

\section{PROBLEM FÖRMULATION}

Let $c(r, z)$ denote the ocean sound speed profile at range $r$ and depth $z$. Consideration will be restricted to single pulse statistics. Therefore, in adopting a time-independent profile model, we have tacitly assumed that the ocean remains "frozen" during the transit time of the pulse. In particular, we shall adopt the representation

$$
c^{-2}(r, z)=c_{0}^{-2}[\zeta(z)+\epsilon \xi(r, z)],
$$

where $c_{0}$ is the channel axis sound speed, $\zeta(z)$ is the term arising from the deterministic profile variation, $\epsilon$ is a small real parameter, and $\xi(r, z)$ is a zero mean, unit-variance random field modeling the sound speed fluctuations.
A point source is assumed to be located at $r=0$, $z=z_{s}$, and is assumed to emit a signal having time de pendence $f_{s}(t)$. Typically, $f_{s}(t)=\exp \left[-t^{2} /\left(2 T_{0}^{2}\right)+i \omega_{0} t\right]$, where $\omega_{0}=2 \pi f_{0}$ denotes the radian carrier frequency. The wave equation for the complex pressure $p(r, z, t)$ then becomes

$\frac{\partial^{2}}{\partial r^{2}} p+r^{-1} \frac{\partial}{\partial r} p+\frac{\partial^{2}}{\partial z^{2}} p-c^{-2} \frac{\partial^{2}}{\partial t^{2}} p=-\frac{\delta(r)}{2 \pi r} \delta\left(z-z_{s}\right) f_{s}$.

In addition to (2), an outgoing wave condition as $r \rightarrow \infty$ as well as suitable boundary conditions at the ocean surface and bottom must be imposed. However, the precise form of these constraints will not be important for the discussion that follows.

Let $\omega$ denote radian frequency and $k \equiv \omega / c_{0}$ represent wavenumber relative to the axis sound speed. Let $\hat{p}$ and $\hat{f}_{s}$ denote Fourier transforms, e.g.,

$$
\hat{p}(r, z, k) \equiv(2 \pi)^{-1 / 2} \int_{-\infty}^{\infty} p(r, z, t) e^{-i k c_{0} t} d t .
$$

Then, the transformed version of (2) becomes

$\frac{\partial^{2}}{\partial r^{2}} \hat{p}+r^{-1} \frac{\partial}{\partial r} \hat{p}+\frac{\partial^{2}}{\partial z^{2}} \hat{p}+k^{2}(\zeta+\epsilon \xi) \hat{p}=-\frac{\delta(r)}{2 \pi r} \delta\left(z-z_{s}\right) \hat{f}_{s}$.

We shall first consider (4) for $r>0$ and, subsequently, account for the source by a matching procedure. If the cylindrical spreading factor is removed by defining $\hat{\psi}(r, z, k) \equiv r^{1 / 2} \hat{p}(r, z, k)$, and if the near-field term $\hat{\psi} /$ $\left(4 r^{2}\right)$ is neglected, the equation for $\hat{\psi}$ is

$$
\frac{\partial^{2}}{\partial r^{2}} \hat{\psi}+\frac{\partial^{2}}{\partial z^{2}} \hat{\psi}+k^{2}(\zeta+\epsilon \xi) \hat{\psi}=0, \quad r>0 .
$$

We shall now expand $\hat{\psi}$ in the normal modes of the unperturbed depth-dependent problem. Let $\phi_{n}(z, k)$ and $\beta_{n}^{2}(k)$ denote the (real-valued) $n$th eigenfunction and eigenvalue of the depth-dependent problem. (We ignore the possibility of radiation modes and associated continuous spectrum.) These "trapped-mode" eigenfunctions satisfy the eigenvalue equation

$$
\frac{d^{2}}{d z^{2}} \phi_{n}+k^{2} \zeta(z) \phi_{n}=\beta_{n}^{2} \phi_{n}, \quad n=1,2, \ldots,
$$

together with appropriate boundary conditions and an orthonormality relation, i.e., $\int \phi_{n} \phi_{m} d z=\delta_{m n \cdot}$. At any given frequency (or wavenumber), only a finite number 
of the $\beta_{n}$ are real, say $1 \leqslant n \leqslant N(k)$. These correspond to propagating modes, modes that transport acoustic energy. The remaining infinity of modes are evanescent or cut off. These modes decay exponentially with range; they do not transport energy and will be ignored:

Use of the representation $\hat{\psi}(r, z, k) \equiv \sum_{n} c_{n}(r, k) \phi_{n}(z, k)$ in (5) leads to the following set of coupled mode equations :

$$
\begin{aligned}
& \frac{d^{2}}{d r^{2}} c_{m}(r, k)+\beta_{m}^{2} c_{m}(r, k) \\
& =-\epsilon \sum_{n=1}^{N(k)} \xi_{m n}(r, k) c_{n}(r, k), \quad m=1, \ldots, N(k), \\
& \xi_{m n}(r, k) \equiv \int \xi(r, z) \phi_{m}(z, k) \phi_{n}(z, k) d z,
\end{aligned}
$$

where modal orthonormality has been exploited, and the evanescent modes have been discarded. The rapid phase variation is now eliminated by transforming to "slowly varying" modal coefficients as follows:

$$
\begin{aligned}
& c_{m} \equiv \beta_{m}^{-1 / 2}\left(A_{m} e^{-i \beta_{m} r}+B_{m} e^{i \beta_{m} r}\right), \quad m=1, \ldots, N . \\
& e^{-i \beta_{m} r} \frac{d}{d r} A_{m}+e^{i \beta_{m} r} \frac{d}{d r} B_{m} \equiv 0,
\end{aligned}
$$

When Eqs. (8) are used in (7), a complex $2 N$-dimensional first order linear system of ordinary differential equations is obtained, the dependent variables being $A_{m}(r, k)$ and $B_{m}(r, k), 1 \leqslant m \leqslant N(k)$. Note that $A_{m}$ and $B_{m}$ correspond, respectively, to slowly varying forward and backward propagating modal coefficients; they are constant in range if $\epsilon=0$ (i.e., no random sound speed fluctuations). We shall now assume that backscattering is negligible and set $B_{m}(r, k) \equiv 0$. This forward scattering approximation is commonly adopted; it can be justified for the particular problem of interest. ${ }^{12}$ The resulting system of equations for the forward-propagating $A_{m}$ modal coefficients then becomes

$$
\begin{aligned}
\frac{d}{d r} A_{m}(r, k)= & -i \epsilon \sum_{n=1}^{N(k)} \exp \left\{i\left[\beta_{m}(k)-\beta_{n}(k)\right] r\right\} \\
& \times \eta_{m n}(r, k) A_{n}(r, k), \\
\eta_{m n}(r, k) \equiv & k^{2} /\left\{2\left[\beta_{m}(k) \beta_{n}(k)\right]^{1 / 2}\right\} \\
& \times \xi_{m n}(r, k), \quad m=1, \ldots, N(k) .
\end{aligned}
$$

$$
\begin{aligned}
\left\langle p(r, z, t) p^{*}\left(r^{\prime}, z^{\prime}, t^{\prime}\right)\right\rangle= & \frac{c_{0}^{2}}{2 \pi\left(r r^{\prime}\right)^{1 / 2}} \int_{-\infty}^{\infty} \int_{-\infty}^{\infty} \sum_{n=1}^{N(k)} \sum_{n^{\prime}=1}^{\alpha\left(k^{\prime}\right)}\left\langle A_{n}(r, k) A_{n^{\prime}}^{*}\left(r^{\prime}, k^{\prime}\right)\right\rangle \\
& \times \phi_{n}(z, k) \phi_{n^{\prime}}\left(z^{\prime}, k^{\prime}\right) \frac{\exp \left\{i\left[c_{0}\left(k t-k^{\prime} t^{\prime}\right)-\left(\beta_{n}(k) r-\beta_{n^{\prime}}\left(k^{\prime}\right) r^{\prime}\right)\right]\right\}}{\left[\beta_{n}(k) \beta_{n^{\prime}}\left(k^{\prime}\right)\right]^{1 / 2}} d k d k^{\prime}
\end{aligned}
$$

We shall extend the summations in (15) to infinity by setting $\phi_{n}(z, k) \equiv 0$ for $n>N(k)$. Since (15) will be evaluated in the asymptotic limit of long ranges and weak random inhomogeneities, the variables $r$ and $r^{\prime}$ (hence, also $c_{0} t$ and $\left.c_{0} t^{\prime}\right)$ will be assumed to be large. Even at such long ranges, however, the second moment of the modal coefficients $\left\langle A_{n}(r, k) A_{n^{\prime}}^{*}\left(r^{\prime}, k^{\prime}\right)\right\rangle$ undergoes relatively slow variation [c.f. (9)]. Therefore the Method of Stationary Phase ${ }^{14}$ can be used to evaluate (15); the result is
To complete the specification of (9), initial conditions $A_{m}(0, k)$ must be specified. These conditions, dictated by the source, will be obtained by a matching procedure. The basic underlying assumption will be that the guration before the random inhomogeneities can exert

In the absence of random perturbations (i.e., $\epsilon=0$ ), an outgoing wave solution for $\hat{\psi}$ can be obtained by separation of variables. The resulting series solution

$$
\hat{\psi}(r, z, k)=\sum_{n=1}^{\infty} \alpha_{n}(k) r^{1 / 2} H_{0}^{(2)}\left[\beta_{n}(k) r\right] \phi_{n}(z, k) .
$$

The orthonormality of the mode functions $\phi_{n}$ and the fact that ${ }^{13}$

$$
\left(\frac{d^{2}}{d r^{2}}+r^{-1} \frac{d}{d r}+\beta_{n}^{2}\right) H_{0}^{(2)}\left(\beta_{n} r\right)=-i \frac{4}{2 \pi r} \delta(r),
$$

imply that $\alpha_{n}(k)=-(i / 4) \phi_{n}\left(z_{s}, k\right) \hat{f}_{s}(k), n=1,2, \ldots$ Use of the farfield form of the Hankel function, $H_{0}^{(2)}\left(\beta_{n} \gamma\right)$ $\sim\left(2 / \pi \beta_{n} r\right)^{1 / 2} \exp \left[-i\left(\beta_{n} r-\pi / 4\right)\right]$, and neglect of the evanescent modes lead to the asymptotic representa tion

$$
\begin{aligned}
\hat{\psi}(r, z, k) \sim & \sum_{n=1}^{N(k)}\left[-\frac{i}{4} e^{i(r / 4)}\left(\frac{2}{\pi \beta_{n}}\right)^{1 / 2} \phi_{n}\left(z_{s}, k\right)\right. \\
& \left.\times \hat{f}_{s}(k)\right] e^{-i \beta_{n} r} \phi_{n}(z, k) .
\end{aligned}
$$

On the other hand, the outgoing farfield approximate solution in the presence of random inhomogeneities was found to be

$$
\hat{\psi}(r, z, k) \sim \sum_{n=1}^{N(k)} \frac{A_{n}(r, k)}{\left(\beta_{n}\right)^{1 / 2}} e^{-i \beta_{n} r} \phi_{n}(z, k) .
$$

The matching will simply consist of requiring that (12) and (13) agree for "small $r$ ", i.e., in the farfield, but before stochastic effects appreciably develop. In particular, we shall demand that

$$
\begin{aligned}
A_{n}(0, k)= & -(i / 4)(2 / \pi)^{1 / 2} e^{i(r / 4)} \\
& \times \phi_{n}\left(z_{s}, k\right) \hat{f}_{s}(k), \quad n=1, \ldots, N(k) .
\end{aligned}
$$

Equations (9) and (14) define the initial value problem for the forward propagating modal coefficients $A_{n}(r, k)$. The quantity of ultimate interest is the mutual cor. herence function for the complex pressure 


$$
\begin{aligned}
\left\langle p(r, z, t) p^{*}\left(r^{\prime}, z^{\prime}, t^{\prime}\right)\right\rangle \sim & \frac{c_{0}^{2}}{r r^{\prime}} \sum_{n=1}^{\infty} \sum_{n^{\prime}=1}^{\infty}\left\langle A_{n}\left(r, \kappa_{n}\right) A_{n^{\prime}}^{*}\left(r^{\prime}, \kappa_{n^{\prime}}\right)\right\rangle \phi_{n}\left(z, \kappa_{n}\right) \phi_{n^{\prime}}\left(z^{\prime}, \kappa_{n^{\prime}}\right) \\
& \times \frac{\exp \left\{i\left[c_{0}\left(\kappa_{n} t-\kappa_{n^{\prime}} t^{\prime}\right)-\left(\beta_{n}\left(\kappa_{n}\right) r-\beta_{n^{\prime}}\left(\kappa_{n^{\prime}}\right) r^{\prime}\right)\right]\right\}}{\left[\beta_{n}\left(\kappa_{n}\right) \beta_{n^{\prime}}\left(\kappa_{n^{\prime}}\right)\left|\ddot{\beta}_{n}\left(\kappa_{n}\right) \ddot{\beta}_{n^{\prime}}\left(\kappa_{n^{\prime}}\right)\right|\right]^{1 / 2}},
\end{aligned}
$$

where $\kappa_{n}$ denotes the stationary wavenumber [i.e., $\left.(d / d k) \beta_{n}\left(\kappa_{n}\right)=c_{0} t / r\right]$ and $\ddot{\beta}_{n} \equiv\left(d^{2} / d k^{2}\right) \beta_{n}$. In principle, an explicit dispersion relation can be obtained by expressing the wavenumber $\kappa_{n}$ as a function of $c_{0} t / r$. Therefore, as (16) indicates, we must determine the asymptotic behavior of the second moment $\left\langle A_{n}\left(r, \kappa_{n}\right) A_{n^{\prime}}^{*}\left(r^{\prime}, k_{n^{\prime}}\right)\right\rangle$, where $\kappa_{n}=\kappa_{n}\left(c_{0} t / r\right)$.

Equation (16) has the ostensible structure of a single eigenray expansion, expressed in terms of normal modes. In Sec. II, however, we shall show that (16) is best viewed as a sum of two terms or expansions, since the second moments appearing in that equation will evolve with range very differently in the two cases $n=n^{\prime}$ and $n \neq n^{\prime}$ [c.f.(22)]. One of these terms will correspond to the random spreading and attenuation of an underlying deterministic eigenray structure; individual contributions arising from this term will spread and decay in strength as range increases. The second term, however, will initially be zero and will grow due to the random fluctuations, reaching an eventual limiting configuration as range (and hence the cumulative impact of the small random perturbations) continues to increase.

\section{ASYMPTOTIC BEHAVIOR OF THE SECOND MOMENTS}

Since the asymptotic limit of interest involves long propagation ranges, the sum variables $R \equiv \frac{1}{2}\left(r+r^{\prime}\right)$ and $c_{0} T \equiv\left(c_{0} / 2\right)\left(t+t^{\prime}\right)$ are large. On the other hand, the difference variables $r-r^{\prime}$ and $c_{0}\left(t-t^{\prime}\right)$ remain relatively small since they are constrained essentially by the space-time extent of the propagating pulse. Recall that $\epsilon^{2}$ is a small parameter scaling the variance of the relative sound speed fluctuations. The particular asymptotic limit to be considered will entail $O\left(\epsilon^{-2}\right)$ ranges; therefore let $\rho \equiv \epsilon^{2} R$ and $\tau \equiv \epsilon^{2} T$.

Note that the large "center-of-mass" range $R$ will play the dominant role in determining the asymptotic behavior of $\left\langle A_{n}\left(r, \kappa_{n}\right) A_{n^{\prime}}^{*}\left(r^{\prime}, \kappa_{n^{\prime}}\right)\right\rangle$, i.e., the relative range offsets are negligible. Therefore,

$$
\left\langle A_{n}\left(r, \kappa_{n}\right) A_{n^{\prime}}^{*}\left(r^{\prime}, \kappa_{n^{\prime}}\right)\right\rangle \cong\left\langle A_{n}\left(R, \kappa_{n}\right) A_{n^{\prime}}^{*}\left(R, \kappa_{n^{\prime}}\right)\right\rangle .
$$

Note further that $c_{0} t / r \cong c_{0} t^{\prime} / r^{\prime} \cong c_{0} \tau / \rho$. Therefore the dependence of the stationary wavenumber $\kappa_{n}$ upon range and time involves a slowly varying (quasi-static) ratio.

Consider first the case where the dependence of stationary wavenumber upon range and time is assumed to be truly quasi-static, i.e., $\kappa_{n}$ and $\kappa_{n^{\circ}}$ are treated as fixed constants. In this case, the asymptotic analysis has already been developed. ${ }^{10-12}$ As $\epsilon \rightarrow 0$ and $R \rightarrow \infty$, with $\rho=\epsilon^{2} R$ remaining $O(1)$, the second moment $\left\langle A_{n}\left(\rho / \epsilon^{2} ; \kappa_{n}\right) A_{n^{\prime}}^{*}\left(\rho / \epsilon^{2} ; \kappa_{n^{\prime}}\right)\right\rangle$ converges to a correspon- ding second moment of a Markov diffusion process. The principal underlying physical assumptions are that the random field $\xi$ possess a mixing or asymptotic independence property and be wide-sense stationary in range. Both of these assumptions are compatible with models of ocean sound speed fluctuations.

The importance of this asymptotic result lies in the fact that expectations of functions of a Markov diffusion process can be evaluated by solving partial differential equations (Kolmogorov equations). Moreover, in this case where the functions involved are polynomials (i.e., moments) and the original stochastic Eqs. (9) are linear, the computations further simplify to the level of linear ordinary differential equations.

In order to describe the asymptotic behavior of the second moments, the following two cases must be distinguished:

(a) $n=n^{\prime}$ :

In this case,

$$
\left\langle A_{n}\left(\rho / \epsilon^{2}, \kappa_{n}\right) A_{n}^{*}\left(\rho / \epsilon^{2}, \kappa_{n}\right)\right\rangle=\left\langle\left|A_{n}\left(\rho / \epsilon^{2}, \kappa_{n}\right)\right|^{2}\right\rangle
$$

essentially represents the expected power in the $n$th mode at wave number $\kappa_{n}$ and range $\rho / \epsilon^{2}$. Let the asymptotic behavior of this second moment be denoted by $w_{n}\left(\rho, \kappa_{n}\right)$; more generally, let $w_{l}\left(\rho, \kappa_{n}\right)$ denote the asymptotic behavior of the expected power in the $l$ th mode at wavenumber $\kappa_{n}$. Then, the set of functions $\left\{w_{l}\left(\rho, \kappa_{n}\right)\right\}_{l=1}^{N\left(\kappa_{n}\right)}$ obeys the following coupled power equations ${ }^{6,11}$ :

$$
\begin{gathered}
\frac{d}{d \rho} w_{l}\left(\rho, \kappa_{n}\right)=2 \sum_{m=1}^{N\left(\kappa_{n}\right)} a_{l m}\left(\kappa_{n}\right)\left[w_{m}\left(\rho, \kappa_{n}\right)-w_{l}\left(\rho, \kappa_{n}\right)\right] \\
w_{l}\left(0, \kappa_{n}\right)=\left[\left|\hat{f}_{s}\left(\kappa_{n}\right)\right|^{2} / 8 \pi\right] \phi_{l}^{2}\left(z_{s}, \kappa_{n}\right) \\
{[\text { cf. }(14)], 1 \leqslant l \leqslant N\left(\kappa_{n}\right)}
\end{gathered}
$$

where

$$
\begin{aligned}
a_{l m}\left(\kappa_{n}\right) \equiv & \int_{0}^{\infty}\left\langle\eta_{l m}\left(r, \kappa_{n}\right) \eta_{l m}\left(0, \kappa_{n}\right)\right\rangle \\
& \times \cos \left\{\left[\beta_{l}\left(\kappa_{n}\right)-\beta_{m}\left(\kappa_{n}\right)\right] r\right\} d r,
\end{aligned}
$$

and $\eta_{l m}$ is defined by (9).

(b) $n \neq n^{\prime}:$

Let the asymptotic behavior of

$$
\left\langle A_{n}\left(\rho / \epsilon^{2}, \kappa_{n}\right) A_{n^{\prime}}^{*}\left(\rho / \epsilon^{2}, \kappa_{n^{0}}\right)\right\rangle
$$

be denoted by $w_{n n^{\prime}}\left(\rho, \kappa_{n}, \kappa_{n^{\prime}}\right)$. One can show that 


$$
\begin{gathered}
\frac{d}{d \rho} w_{n n^{\prime}}\left(\rho, \kappa_{n}, \kappa_{n^{\prime}}\right)=-\Lambda\left(\kappa_{n}, \kappa_{n^{\prime}}\right) w_{n n^{\prime}}\left(\rho, \kappa_{n}, \kappa_{n^{\prime}}\right), \\
w_{n n^{\prime}}\left(0, \kappa_{n}, \kappa_{n^{\prime}}\right)=\left[\hat{f}_{s}\left(\kappa_{n}\right) \hat{f}_{s}^{*}\left(\kappa_{n^{\prime}}\right) / 8 \pi\right] \\
\times \phi_{n}\left(z_{s}, \kappa_{n}\right) \phi_{n^{\prime}}\left(z_{s}, \kappa_{n^{\prime}}\right),
\end{gathered}
$$

where

$$
\begin{aligned}
\Lambda\left(\kappa_{n}, \kappa_{n^{\prime}}\right) \equiv & \sum_{m=1}^{N\left(\kappa_{n}\right)} a_{m n^{\prime} m n}^{*}\left(\kappa_{n}\right) \\
& +\sum_{m^{\prime}=1}^{\left.N \kappa_{n^{\prime}}^{\prime}\right)} a_{m^{\prime} n^{\prime}, m^{\prime} n^{\prime}}\left(\kappa_{n^{\prime}}\right)-\tilde{a}\left(\kappa_{n}, \kappa_{n^{\prime}}\right), \\
a_{m n, m n}\left(\kappa_{n}\right) \equiv & \int_{0}^{\infty}\left\langle\eta_{m n}\left(r, \kappa_{n}\right) \eta_{m n}\left(0, \kappa_{n}\right)\right\rangle \\
& \times \exp \left\{i\left[\beta_{m}\left(\kappa_{n}\right)-\beta_{n}\left(\kappa_{n}\right)\right] r\right\} d r, \\
\tilde{a}\left(\kappa_{n}, \kappa_{n^{\prime}}\right) \equiv & \int_{0}^{\infty}\left\langle\eta_{m n}\left(r, \kappa_{n}\right) \eta_{n^{\prime} n^{\prime}}\left(0, \kappa_{n^{\prime}}\right)\right. \\
& \left.+\eta_{n^{\prime} n^{\prime}}\left(r, \kappa_{n^{\prime}}\right) \eta_{n n^{\prime}}\left(0, \kappa_{n}\right)\right\rangle d r .
\end{aligned}
$$

Thus as a consequence of (19) we obtain the explicit representation $w_{n n}\left(\rho, \kappa_{n}, \kappa_{n}\right)=w_{n n},\left(0, \kappa_{n}, \kappa_{n}\right)$ $\times \exp \left[-\rho \Lambda\left(\kappa_{n}, \kappa_{n}\right)\right]$.
With regard to Eqs. (17)-(21), the following points should be noted. Range dependence enters into the asymptotic description solely through the scaled range $\rho=\epsilon^{2} R$. Since $\epsilon^{2}$ is a small parameter, the actual propagation range $R$ must become large before significant probabilistic effects emerge. The coupling coefficients $a_{t m}\left(\kappa_{n}\right)$ are basically cosine transforms of correlation functions (i.e., power spectrum evaluations) and thus are nonnegative. One can show that $\operatorname{Re}\left[\Lambda\left(\kappa_{n}, \kappa_{n^{\prime}}\right)\right] \geqslant 0$ so that $w_{n n^{\circ}}$ is an exponentially decaying function of scaled range. On the other hand, the functions $w_{l}\left(\rho, \kappa_{n}\right)$ do not undergo such decay. As a consequence of (17) and the fact that $a_{l m}=a_{m l}$, we obtain the conservation relation

$$
\frac{d}{d \rho} \sum_{i=1}^{N\left(\kappa_{n}\right)} w_{l}\left(\rho, \kappa_{n}\right)=0
$$

Moreover, as scaled range $\rho$ becomes large the solutions of (17) tend toward equipartition, i.e.,

$$
\lim _{\rho \rightarrow \infty} w_{l}\left(\rho, \kappa_{n}\right)=\frac{1}{N\left(\kappa_{n}\right)} \sum_{m=1}^{N\left(\kappa_{n}\right)} w_{m}\left(0, \kappa_{n}\right)
$$

In terms of the functions $w_{l}$ and $w_{n n^{\prime}}$, we can express the mutual coherence function of interest as

$$
\begin{aligned}
\left\langle p(r, z, t) p^{*}\left(r^{\prime}, z^{\prime}, t^{\prime}\right)\right\rangle & \frac{c_{0}^{2}}{R^{2}} \sum_{n=1}^{\infty} \sum_{n^{\prime}=1}^{\infty} w_{n n^{\prime}}\left(\rho, \kappa_{n}, \kappa_{n^{\prime}}\right) \phi_{n}\left(z, \kappa_{n}\right) \phi_{n^{\prime}}\left(z^{\prime}, \kappa_{n^{\prime}}\right) \\
& \times \frac{\exp \left\{i\left[c_{0}\left(\kappa_{n} t-\kappa_{n^{\prime}} t^{\prime}\right)-\left(\beta_{n}\left(\kappa_{n}\right) r-\beta_{n^{\prime}}\left(\kappa_{n^{\prime}}\right) r^{\prime}\right)\right]\right\}}{\left[\beta_{n}\left(\kappa_{n}\right) \beta_{n^{\prime}}\left(\kappa_{n^{\prime}}\right)\left|\ddot{\beta}_{n}\left(\kappa_{n}\right) \ddot{\beta}_{n^{\prime}}\left(\kappa_{n^{\prime}}\right)\right|\right]^{1 / 2}}+\frac{c_{0}^{2}}{R^{2}} \sum_{n=1}^{\infty}\left[w_{n}\left(\rho, \kappa_{n}\right)-w_{m n}\left(\rho, \kappa_{n}, \kappa_{n}\right)\right] \\
& \times \phi_{n}\left(z, \kappa_{n}\right) \phi_{n}\left(z^{\prime}, \kappa_{n}\right) \frac{\exp \left\{i\left[c_{0} \kappa_{n}\left(t-t^{\prime}\right)-\beta_{n}\left(\kappa_{n}\right)\left(r-r^{\prime}\right)\right]\right\}}{\beta_{n}\left(\kappa_{n}\right)\left|\ddot{\beta}_{n}\left(\kappa_{n}\right)\right|} .
\end{aligned}
$$

Recall that the $w_{n n^{\prime}}$ terms appearing in (22) consist essentially of the transmitted signal weighted by an exponential factor arising from the random field. In the next section, we shall consider a specific example and show, using the Poisson Sum Formula, that the double sum in (22) accounts for an initial spreading of the transmitted pulse. The double sum, therefore, will prove to be particularly important in describing the behavior of the propagating signal at relatively small values of scaled range $\rho$. As $\rho$ continues to increase, however, the single sum term in (22) becomes increasingly important.

The factor $\left(w_{n}-w_{m}\right)$ vanishes when $\rho=0$. Moreover, one can show that $\left(w_{n}-w_{n n}\right) \geqslant 0$ for all values of $\rho$. As scaled range increases, this factor increases from an initial value of zero and approaches the value $N^{-1}\left(\kappa_{n}\right)$ $\times \sum_{n \rightarrow 1}^{N\left(K_{n}\right)} w_{n}\left(0, \kappa_{n}\right)$ as $\rho \rightarrow \infty$. The single sum in (22) consequently plays an important role in modeling the behavior of the propagating signal at large values of scaled range. As we shall observe in the next section, this term will evolve to a multiple arrival structure with an algebraically varying envelope.

Thus far the discussion has been based upon the assumption that the stationary wavenumber $\kappa_{n}$ is a fixed constant. In fact, as was previously noted, $\kappa_{n}$ is a function of $c_{0} \tau / \rho$. However, we shall now show that accounting for this dependence does not change the essential features of the prior discussion. Consider first the case $n=n^{\prime}$. The modal power $w_{l}\left(\rho, \kappa_{n}\right)$ is now a function of $\rho$ and $c_{0} \tau / \rho$; we denote this dependence by $w_{l}\left[\rho,\left(c_{0} \tau / \rho\right)\right]$. Then

$$
\frac{\partial}{\partial \rho} w_{l}=\partial_{1} w_{l}-\left(c_{0} \tau / \rho^{2}\right) \partial_{2} w_{l},
$$

where $\partial_{1}, \partial_{2}$ denote partial differentiation with respect to the first and second arguments, respectively. However, $\partial_{1} w_{l}=2 \sum_{m=1}^{\left.N^{(k}\right)} a_{l m}\left(\kappa_{n}\right)\left(w_{m}-w_{l}\right)$ [c.f. (17)] and $\left(c_{0} / \rho\right)$ $\times \partial_{2} w_{l}=(\partial / \partial \tau) w_{l}$. Therefore the counterpart of (17) becomes

$$
\frac{\partial}{\partial \rho} w_{l}+\left(\frac{\tau}{\rho}\right) \frac{\partial}{\partial \tau} w_{l}=2 \sum_{m=1}^{N\left(k_{r}\right)} a_{l m}\left(\kappa_{n}\right)\left(w_{m}-w_{l}\right) .
$$

Similarly, for the case $n \neq n^{\prime}$ we view $\kappa_{n}$ and $\kappa_{n}$, as functions of $c_{0} \tau / \rho$ and obtain the following counterpart of (19),

$$
\frac{\partial}{\partial \rho} w_{n n^{\prime}}+\left(\frac{\tau}{\rho}\right) \frac{\partial}{\partial \tau} w_{n n^{\prime}}=-\Lambda\left(\kappa_{n}, \kappa_{n^{\prime}}\right) w_{n n^{\prime}} .
$$

Equations (24) and (25) must be viewed as outer equations, valid away from $\rho=0$. This is consistent with our prior use of the Method of Stationary Phase, which requires that $\rho$ and $\tau$ be both nonzero. In principle, then, a matching procedure would be needed to connect 
(24) and (25) to the initial data at $\rho=0$. Perhaps the simplest such procedure would be to impose the data at some small positive value of scaled range (i.e., neglect the effect of random fluctuations on some small initial interval of scaled range). The particular cases to be considered in the next section, however, will not require us to specifically address this point.

The quantity of interest, the mutual coherence function of the complex pressure, is thus given by (22), where $w_{n}$ and $w_{n n^{\prime}}$ are now solutions of (24) and (25), respectively. Note, however, that along the characteristics of these first order equations, the ratio $c_{0} \tau / \rho$ remains constant. Therefore the prior discussion applies along the characteristics since wavenumber remains constant along these space-time paths. For example, we can again conclude that

$$
\begin{aligned}
& w_{n n^{\circ}}=\left\{\left[\hat{f}_{s}\left(\kappa_{n}\right) \hat{f}_{s}^{*}\left(\kappa_{n^{\prime}}\right) \phi_{n}\left(z_{s}, \kappa_{n}\right) \phi_{n^{\circ}}\left(z_{s}, \kappa_{n^{\prime}}\right)\right] / 8 \pi\right\} \\
& \times \exp \left[-\rho \Lambda\left(\kappa_{n}, \kappa_{n^{\circ}}\right)\right] .
\end{aligned}
$$

Now, however, modal dispersion is accounted for by the fact that $\kappa_{n}$ is a function of $c_{0} \tau / \rho$.

\section{INTERPRETATION OF THE RESULTS}

Since the theory developed is based upon a coupled mode analysis, it will be most useful in modeling experiments where the number of modes involved is small (e.g., volume scattering of low-frequency pulses or configurations where the interaction of bottom-bounce modes through rough surface or sediment scattering is dominant). Note in particular that if bottom effects are dominant and volume scattering can be ignored, the random coupling problem will involve only that subset of modes which interact significantly with the bottom.

For the present discussion, however, to illustrate the content of (22), we shall consider the volume scattering of a short pulse in the two limiting cases where only the double or single sums in (22) are important. We shall adopt a model deterministic profile of the type discussed by Hirsch and Carter ${ }^{15}$; referring to (1), we set

$$
\zeta(z)=1-|\alpha z|^{5}, \quad 1<\delta<2,
$$

where the channel axis is assumed to lie at $z=0$. Restricting $\delta$ to values between 1 and 2 leads to the more realistic situation where off-axis arrivals will precede the direct arrival if source and receiver lie on the channel axis. Note that one could, with virtually no added difficulty, assume a piecewise profile of the type given by (26), i.e., adopt different values of $\alpha$ for positive and negative values of $z$.
Using the WKB approximation for the normal modes, we obtain ${ }^{8}$ :

$$
\phi_{n}(z, k) \cong\left\{\begin{array}{l}
\frac{\cos \left(\theta_{n}(z, k)-\pi / 4\right)}{2\left[\gamma_{n}(z, k) \nu_{n}(k)\right]^{1 / 2}}, \quad z_{l}<z<z_{u}, \\
0, \text { otherwise, }
\end{array}\right.
$$

where $z_{l}$ and $z_{u}$ denote the turning points. (The angle $\theta_{n}$ and the product $\gamma_{n} \nu_{n}$ are specified in (28).) The quantities appearing in (27), as well as the others appearing in (22), must be evaluated at the stationary wavenumber $\kappa_{n}$. For the profile defined by (26), we can conveniently express the quantities of interest in terms of an angle $\psi$ as follows:

$$
\begin{aligned}
& \kappa_{n}=\left(n+\frac{1}{2}\right)\left[\alpha \delta \pi / 2 B\left(\delta^{-1}, \frac{3}{2}\right)\right](\csc \psi)^{1+2 / \sigma}, \\
& \beta_{n}\left(\kappa_{n}\right)=\kappa_{n} \cos \psi, \\
& \theta_{n}\left(z, \kappa_{n}\right)=\left(n+\frac{1}{2}\right) \frac{\pi}{2}\left(1-\frac{\operatorname{sgn}(z)}{B\left(\delta^{-1}, \frac{3}{2}\right)} \int_{0}^{\zeta} X^{1 / \delta}(1-X)^{1 / 2} d X\right), \\
& \zeta \equiv|\alpha z|^{6} \csc ^{2} \psi, \\
& \gamma_{n}\left(z, \kappa_{n}\right) \nu_{n}\left(\kappa_{n}\right) \cong\left[b^{\prime}\left(\delta^{-1}, \frac{1}{2}\right) / \alpha \delta\right](\sin \psi)^{2 / \delta}(1-\zeta)^{1 / 2}, \\
& \left|\beta_{n}\left(\kappa_{n}\right) \ddot{\beta}_{n}\left(\kappa_{n}\right)\right|=\left[\tan ^{2} \psi /(\delta+2)^{2}\right]\left|\delta^{2}-2 \delta \cos ^{2} \psi\right|,
\end{aligned}
$$

where $B(z, w) \equiv \int_{0}^{1} X^{z-1}(1-X)^{w-1} d X$ is the beta function ${ }^{16}$ and the dispersion relation is implicitly given by the equation

$$
\left(c_{0} \tau / \rho\right)=\sec \psi\left\{1-[2 /(\delta+2)] \sin ^{2} \psi\right\} .
$$

As previously noted, we shall assume a transmitted signal of the form

$$
f(t)=\exp \left(-t^{2} / 2 T_{0}^{2}+i \omega_{0} t\right),
$$

so that

$$
\hat{f}_{s}\left(\kappa_{n}\right)=T_{0} \exp \left[-\left(T_{0}^{2} / 2\right)\left(c_{0} \kappa_{n}-\omega_{0}\right)^{2}\right] .
$$

For simplicity, we shall restrict consideration to the case $r=r^{\prime}=R, t=t^{\prime}=T, z=z^{\prime}$, i.e., to a consideration of $\left\langle|p(r, z, t)|^{2}\right\rangle$.

Consider the double sum in (22); recall that

$$
\begin{aligned}
w_{n n^{\prime}}= & (8 \pi)^{-1} \hat{f}_{s}\left(\kappa_{n}\right) \hat{f}_{s}^{*}\left(\kappa_{n^{\prime}}\right) \phi_{n}\left(z_{s}, \kappa_{n}\right) \phi_{n^{\prime}}\left(z_{s}, \kappa_{n^{\prime}}\right) \\
& \times \exp \left[-\rho \Lambda\left(\kappa_{n}, \kappa_{n^{\prime}}\right)\right] .
\end{aligned}
$$

If $\omega_{0} T_{0}$ is sufficiently large (e.g., $\omega_{0} T_{0}>2$ ), both summations can be extended to $-\infty$ with negligible error. The total dependence upon the indices $n$ and $n^{\prime}$ occurs in the product

$$
\begin{gathered}
\hat{f}_{s}\left(\kappa_{n}\right) \hat{f}_{s}^{*}\left(\kappa_{n^{\prime}}\right) \cos \left[\theta_{n}\left(z_{s}, \kappa_{n}\right)-\pi / 4\right] \cos \left[\theta_{n}\left(z, \kappa_{n}\right)-\pi / 4\right] \cos \left[\theta_{n^{\prime}}\left(z_{s}, \kappa_{n^{\prime}}\right)-\pi / 4\right] \\
\times \cos \left[\theta_{n^{\prime}}\left(z, \kappa_{n^{\prime}}\right)-\pi / 4\right] \exp \left[-\rho \Lambda\left(\kappa_{n}, \kappa_{n^{\prime}}\right)\right] \exp \left[i\left(\kappa_{n}-\kappa_{n^{\prime}}\right)\left(c_{0} t-r \cos \psi\right)\right],
\end{gathered}
$$

[cf. (27), (28)]. Noting (28), let $\theta_{n}\left(z, \kappa_{n}\right) \equiv \kappa_{n} \Omega(\zeta)$. Then, if the fourfold product of cosines is written in terms of exponentials, the double sum in (22) decomposes into 16 double sums, each having the form

$$
\begin{aligned}
S \equiv & {\left[\frac{\alpha \delta(\delta+2) c_{0} T_{0} \cos \psi}{32 R B\left(\delta^{-1}, \frac{1}{2}\right)}\right]^{2} \frac{(\csc \psi)^{2+4 / 6} \exp \left[-i(\pi / 4)\left(\epsilon_{1}+\epsilon_{2}-\epsilon_{3}-\epsilon_{4}\right)\right]}{2 \pi(1-\zeta)\left(1-\zeta_{s}\right)\left|\delta^{2}-2 \delta \cos ^{2} \psi\right|} \sum_{n=-\infty}^{\infty} \sum_{n^{\prime}=-\infty}^{\infty} \exp \left[-\left(T_{0}^{2} / 2\right)\left(c_{0} \kappa_{n}-\omega_{0}\right)^{2}\right.} \\
& \left.-\left(T_{0}^{2} / 2\right)\left(c_{0 \kappa_{n^{\prime}}}-\omega_{0}\right)^{2}-\rho \Lambda\left(\kappa_{n}, \kappa_{n^{\prime}}\right)\right] \exp \left\{i \kappa_{n}\left[c_{0} t-\operatorname{rcos} \psi+\epsilon_{1} \Omega(\zeta)+\epsilon_{2} \Omega\left(\zeta_{s}\right)\right]-i \kappa_{n^{\prime}}\left[c_{0} t-\operatorname{rcos} \psi+\epsilon_{3} \Omega(\zeta)+\epsilon_{4} \Omega(\zeta s)\right]\right\},
\end{aligned}
$$


where $\epsilon_{i}= \pm 1, i=1, \ldots, 4$, and the $2^{4}$ possibilities generate the 16 terms.

To complete the specification of (30), one must determine $\Lambda\left(\kappa_{n}, \kappa_{n}\right)$. This requires a specification of the sound speed fluctuation correlation function $\left\langle\epsilon(r, z) \epsilon\left(r^{\prime}, z^{\prime}\right)\right\rangle[\mathrm{cf} .(1)]$. For parameters typical of the ocean regime (i.e., horizontal and vertical correlation lengths on the order of $6 \mathrm{~km}$ and $240 \mathrm{~m}$, respectively), $\Lambda$ has the approximate form

$$
\Lambda\left(\kappa_{n}, \kappa_{n^{0}}\right) \cong\left(\kappa_{n}^{2}+\kappa_{n^{\prime}}^{2}\right) A+\left(\kappa_{n}-\kappa_{n^{0}}\right)^{2} B,
$$

where $A$ and $B$ are real positive functions of $\csc \psi$ and the correlation lengths. In (31), the imaginary part of $\Lambda\left(\kappa_{n}, \kappa_{n^{\prime}}\right)$ has been ignored since one can argue that its relative effect is weak.

Having specified $\Lambda\left(\kappa_{n}, \kappa_{n^{\prime}}\right)$ by (31), we can use the Poisson Sum Formula ${ }^{17}$ to recast (30) into an equivalent double sum having a more direct physical interpretation in terms of ray arrivals. Specifically, we use the relation

$$
\begin{aligned}
& \sum_{n=-\infty}^{\infty} \sum_{n^{\prime}=-\infty}^{\infty} \hat{g}\left(n+\frac{1}{2}, n^{\prime}+\frac{1}{2}\right) \exp \left\{i\left[\left(n+\frac{1}{2}\right) \chi-\left(n^{\prime}+\frac{1}{2}\right) \chi^{\prime}\right]\right\} \\
& =2 \pi \sum_{m=-\infty}^{\infty} \sum_{m^{\prime}=-\infty}^{\infty}(-1)^{m-m^{\prime}} g\left(2 \pi m+\chi, 2 \pi m^{\prime}+\chi^{\prime}\right), \\
& g\left(\chi, \chi^{\prime}\right) \equiv \frac{1}{2 \pi} \int_{-\infty}^{\infty} \int_{-\infty}^{\infty} \hat{g}\left(k, k^{\prime}\right) e^{i\left(k x-k^{\prime} x^{\prime}\right)} d k d k^{\prime} .
\end{aligned}
$$

Applying (32) to (30), with $\Lambda$ given by (31), enables us to rewrite $(30)$ in the form

$$
\begin{aligned}
S=K \sum_{m=-\infty}^{\infty} \sum_{m^{\prime}=-\infty}^{\infty}(-1)^{m-m^{\prime}} \exp [ & -\frac{\left(\gamma+\gamma^{\prime}\right)^{2}}{4 \sigma_{1}^{2}}-\frac{\left(\gamma-\gamma^{\prime}\right)^{2}}{4 \sigma_{2}^{2}} \\
& \left.+i\left(\frac{u \omega_{0} c_{0} T_{0}^{2}}{\sigma_{2}^{2}}\right)\left(\gamma-\gamma^{\prime}\right)\right],
\end{aligned}
$$

where

$$
\begin{aligned}
& a \equiv \kappa_{n} /\left(n+\frac{1}{2}\right)=\left[\alpha \delta \pi / 2 B\left(\delta^{-1}, \frac{3}{2}\right)\right](\csc \psi)^{1+2 / \delta}, \\
& \gamma \equiv 2 \pi m+a\left[c_{0} t-\operatorname{rcos} \psi+\epsilon_{1} \Omega(\zeta)+\epsilon_{2} \Omega\left(\zeta_{s}\right)\right], \\
& \gamma^{\prime} \equiv 2 \pi m^{\prime}+a\left[c_{0} t-\operatorname{rcos} \psi+\epsilon_{3} \Omega(\zeta)+\epsilon_{4} \Omega\left(\zeta_{s}\right)\right], \\
& \sigma_{1}^{2} \equiv a^{2}\left[\left(c_{0} T_{0}\right)^{2}+2 A \rho\right], \\
& \sigma_{2}^{2} \equiv a^{2}\left[\left(c_{0} T_{0}\right)^{2}+2(A+2 B) \rho\right], \\
& K \equiv\left(\frac{B\left(\delta^{-1}, \frac{3}{2}\right) \cos \psi}{16 \pi R B\left(\delta^{-1}, \frac{1}{2}\right)}\right)^{2} \frac{\exp \left[-i(\pi / 4)\left(\epsilon_{1}+\epsilon_{2}-\epsilon_{3}-\epsilon_{4}\right)\right]}{(1-\zeta)\left(1-\zeta_{s}\right)\left|\delta^{2}-2 \delta \cos ^{2} \psi\right|} \\
& \quad \quad \exp \left\{-2 A \rho\left(\omega_{0} T_{0}\right)^{2} /\left[\left(c_{0} T_{0}\right)^{2}+2 A \rho\right]\right\} \\
& \times \frac{\left\{\left[1+2 A \rho /\left(c_{0} T_{0}\right)^{2}\right]\left[1+2(A+2 B) \rho /\left(c_{0} T_{0}\right)^{2}\right]\right\}^{1 / 2}}{\{[1+2 B} .
\end{aligned}
$$

Consider now a single eigen ray ${ }^{15}$ associated with (33). Let $t_{0}$ (as well as $\psi_{0}, \zeta_{0}, \zeta_{s_{0}}$, and $a_{0}$ ) denote the arrival time of this ray. Thus for a particular range, source, and receiver configuration, we assume that there exist values of $m, \epsilon_{1}$ and $\epsilon_{2}$ such that $2 \pi m+a_{0}\left[c_{0} t_{0}-r \cos \dot{\psi}_{0}\right.$ $\left.+\epsilon_{1} \Omega\left(\zeta_{0}\right)+\epsilon_{2} \Omega\left(\zeta_{s_{0}}\right)\right]=0$. The contribution to $\left\langle|p(r, z, t)|^{2}\right\rangle$ due to this eigenray then becomes

$$
\begin{gathered}
K \exp \left(-1 / 4 \sigma_{1}^{2}\left\{a\left[c_{0} t-\mathrm{r} \cos \psi+\epsilon_{1} \Omega(\zeta)+\epsilon_{2} \Omega\left(\zeta_{s}\right)\right]\right.\right. \\
-a_{0}\left[c_{0} t_{0}-\mathrm{rcos} \psi_{0}+\epsilon_{1} \Omega\left(\zeta_{0}\right)+\epsilon_{2} \Omega\left(\zeta_{s_{0}}\right]^{2}\right) .
\end{gathered}
$$

If we neglect dispersion (i.e., set $\psi=\psi_{0}$, so that $a=a_{0}$, $\zeta=\zeta_{0}$, and $\left.\zeta_{s}=\zeta_{s_{0}}\right)$, (34) reduces to $K_{0} \exp \left\{-\left(t-t_{0}\right)^{2} /\right.$ $\left.\left[T_{0}^{2}+2\left(A_{0}+2 B_{0}\right) \rho / c_{0}^{2}\right]\right\}$, where $K_{0}, A_{0}$, and $B_{0}$ denote the evaluation of these terms at $\psi=\psi_{0}$. In this case, the contribution reduces to a Gaussian envelope centered at the ray arrival time and spread by the random inhomogeneities. As one would expect, the spreading increases as range increases. In general, however, as (34) indicates, the interplay of the deterministic profile and the random inhomogeneities is more involved. For contributions arising from small-angle eigenrays, the approximation

$$
\frac{c_{0} t}{r}=\frac{c_{0} \tau}{\rho} \cong 1-\left(\frac{2-\delta}{2+\delta}\right) \frac{\psi^{2}}{2}
$$

[cf. (29)] can be used to recast (34) as an explicit function of time.

As range increases, the random inhomogeneities tend to diffuse or flatten the contribution from any given eigenray. At the same time, however, the number of rays contributing to the total acoustic field at the receiver will generally increase. Thus the ultimate contribution to $\left\langle|p(r, z, t)|^{2}\right\rangle$ by the double sum in (22) will depend upon how this tradeoff evolves as range increases.

With increasing range, the single sum in (22) assumes increasing importance. We shall consider this term for large values of scaled range $\rho$; in that case, noting (21), (27), and (28),

$$
\begin{aligned}
w_{n}-w_{n n} & \cong \frac{1}{N\left(\kappa_{n}\right)} \sum_{m=1}^{N\left(\kappa_{n}\right)} \frac{\left|\hat{f}_{s}\left(\kappa_{n}\right)\right|^{2}}{8 \pi} \phi_{m}^{2}\left(z_{s}, \kappa_{n}\right) \\
& \cong \frac{\left|\hat{f}_{s}\left(\kappa_{n}\right)\right|^{2}}{64 \pi \gamma_{n}\left(z_{s}, \kappa_{n}\right) \nu_{n}\left(\kappa_{n}\right)} .
\end{aligned}
$$

In arriving at (35), we have assumed that scaled range is sufficiently large that $w_{n n}$ has attenuated to a negligible level and that equipartition among the $w_{n}$ has been essentially achieved. The WKB mode functions have been used; note in particular that $\gamma_{m} \nu_{m}$ is independent of $m$. Lastly, we have assumed that $\mid N^{-1}\left(\kappa_{n}\right) \sum_{m=1}^{N\left(\kappa_{n}\right)}$ $\times \sin 2 \theta_{m} \mid \ll 1$. Using (35) and assuming that source and receiver lie on the channel axis, the single sum in (22) (with $r=r^{\prime}=R, t=t^{\prime}=T, z=z^{\prime}=0$ ) becomes [cf. (28) and (33)],

$$
\begin{aligned}
S_{1} & =\left(\frac{c_{0} T_{0}(\delta+2) B\left(\delta^{-1}, \frac{3}{2}\right)}{8 \pi R B\left(\delta^{-1}, \frac{1}{2}\right) \sec \psi}\right)^{2} \frac{1}{\pi\left|\delta^{2}-2 \delta \cos ^{2} \psi\right|} \\
& \times \sum_{n=1}^{\infty} a^{2} \exp \left[-T_{0}^{2}\left(\left(n+\frac{1}{2}\right) c_{0} a-\omega_{0}\right)^{2}\right] .
\end{aligned}
$$

Consider the term $a^{2} \exp \left\{-T_{0}^{2}\left[\left(n+\frac{1}{2}\right) c_{0} a-\omega_{0}\right]^{2}\right\}$. It achieves a maximum value proportional to $\left(n+\frac{1}{2}\right)^{2}$ at a value of $a$-call it $a_{n}$-that is proportional to $\left(n+\frac{1}{2}\right)^{-1}$. Recall that $a \sim(\csc \psi)^{1+2 / 6}$ and that for small values of $\psi$, the dispersion relation (29) can be approximated by

$$
\frac{c_{0} t}{r} \cong 1-\left(\frac{2-\delta}{2+\delta}\right) \frac{\psi^{2}}{2} .
$$

Therefore the larger values of $n$ correspond to the earlier arrivals (since $1<\delta<2$ ).

Using these ideas, the following crude physical inter- 


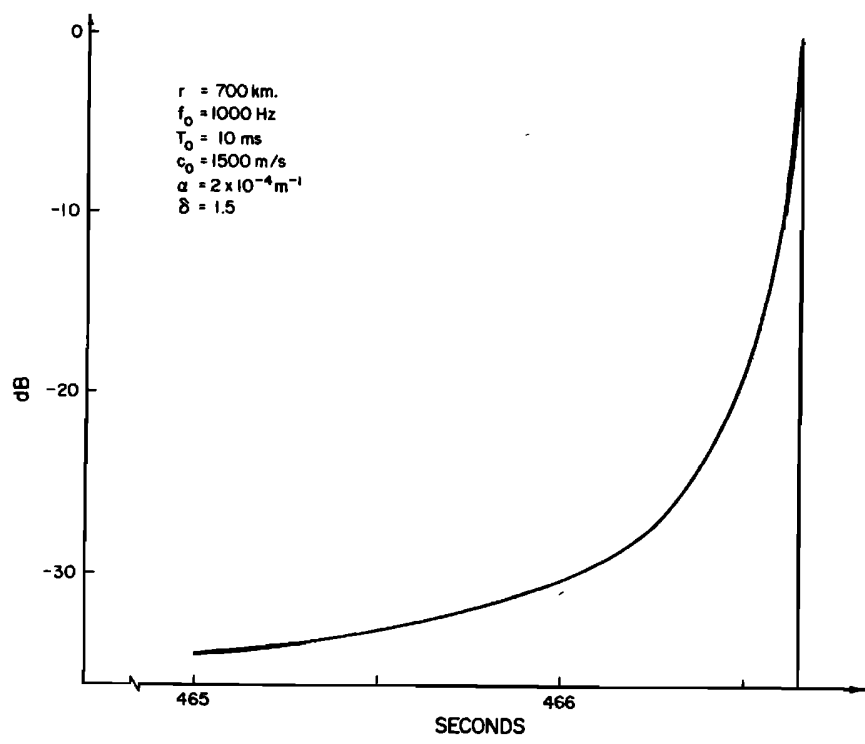

FIG. 1. Normalized envelope of $S_{1}$ (dB) versus time (s).

pretation can be given to $S_{1}$. It consists of a set of arrivals (finite in number since $\csc \psi \geqslant 1$ ), with the earliest being the weakest. As time increases (i.e., $n$ decreases), the arrivals have an envelope that grows algebraically, achieving the largest value at the last arrival. Figure 1 shows the envelope of the sum $S_{1}$ (normalized to $0 \mathrm{~dB}$ ) for a representative set of parameter values. To obtain the total received signal, one would have to superpose the ray arrivals corresponding to the double sum term in (22). In that case, a picture would emerge that is consistent with experimental observations of the signal received from a SOFAR bomb at long range..$^{15}$

\section{CONCLUSIONS}

An asymptotic expression for the mutual coherence function of the complex pressure has been derived for the case of single pulse propagation [c.f. (22)]. The physical content of this expression has been assessed for volume scattering in two limiting cases; in these cases the predictions of the theory seem compatible with experimental observations. However, for the important intermediate case where both terms in (22) are important and many modes are involved, the result in its present form is unwieldy and additional simplification will be necessary to make the expression tractable.

In its present form (22) seems most suited for analyzing pulse propagation experiments wherein the number of randomly coupled modes is moderate (e.g., the volume scattering of low-frequency pulses and/or the sediment scattering of bottom-penetrating modes). Recent experiments ${ }^{18}$ involving sediment scattering have observed multimode arrivals with roughly equal energy content.

\section{ACKNOWLEDGMENTS}

The work was performed under US Office of Naval Research Contract No. N0014-76-C-0056. Attendance at the Seminar on Bottom Effects in Underwater Sound Propagation (Miami, April 1979) proved very helpful in motivating this work.

${ }^{1}$ S. M. Flatté, R. Dashen, W. H. Munk, and F. Zachariasen, "Sound Transmission Through a Fluctuating Ocean," Tech. Rep. JSR-76-39, Stanford Research Institute (1977).

${ }^{2}$ F. Dyson, W. H. Munk, and B. Zetler, "Interpretation of Multipath Scintillations Eleuthera to Bermuda in Terms of Internal Waves and Tides," J. Acoust. Soc. Am. 59, 1121-1133 (1976).

${ }^{3}$ W. H. Munk and F. Zachariasen, "Sound Transmission through a Fluctuating Stratified Ocean-Theory and Observation," J. Acoust. Soc. Am. 59, 818-838 (1976).

${ }^{4} J$. B. Keller and J. S. Papadakis, Wave Propagation and Underwater Acoustics, Lecture Notes in Physics, Vol. 70 (Springer Verlag, New York, 1977).

${ }^{5}$ S. M. Flatté and F. D. Tappert, "Calculation of the Effect of Internal Waves on Oceanic Sqund Transmission," J. Acoust. Soc. Am. 58, 1151-1159 (1975).

${ }^{6}$ L. B. Dozier and F. D. Tappert, "Statistics of Normal Mode Amplitudes in a Random Ocean. I. Theory," J. Acoust. Soc. Am. 63, 353-365 (1978).

${ }^{7}$ L. B. Dozier and F. D. Tappert, "Statistics of Normal Mode Amplitudes in a Random Ocean. II. Computations," J. Acoust. Soc. Am. 64, 533-547 (1978).

${ }^{8}$ R. P. Porter, "Transmission and Reception of Transient Signals in a SOFAR Channel," J. Acoust. Soc. Am. 54, 10811091 (1973).

${ }^{9}$ R. P. Porter and H. D. Leslie, "Energy Evaluation of WideBand SOFAR Transmission," J. Acoust. Soc. Am. 58, 812822 (1975).

${ }^{10} \mathrm{G}$. C. Papanicolaou and W. Kohler, "Asymptotic Theory of Mixing Stochastic Ordinary Differential Equations," Comm. Pure Appl. Math. 27, 641-668 (1974).

${ }^{11}$ Reference 4, Chap. IV.

${ }^{12}$ G. Papanicolaou, P. Chow, and W. Kohler, Proc. Workshop on Wave Propagation through Random Media (North-Holland, New York), to be published.

${ }^{13}$ Reference 4, Chap. II.

${ }^{14} \mathrm{~N}$. Bleistein and R. Handelsman, Asymptotic Evaluation of Integrals (Holt, Rinehart and Winston, New York, 1975).

${ }^{15}$ P. Hirsch and A. H. Carter, "Mathematical Models for the Prediction of SOFAR Propagation Effects," J. Acoust. Soc. Am. 37, 90-94 (1965).

${ }^{16}$ M. Abramowitz and I. A. Stegun, Handbook of Mathematical Functions (Dover, New York, 1970).

${ }^{17}$ G. Carrier, M. Krook, and C. E. Pearson, Functions of a Complex Variable (McGraw-Hill, New York, 1966).

${ }^{18} \mathrm{~A}$. Ellinthorpe, "Some Propagation Statistics Attributable to Bottom Interactions, " lecture presented at Seminar on Bottom Effects in Underwater Sound Propagation (Miami, April 1979). 